RoboCup 2007: Robot WorldCup XI, Lecture Notes in Artificial Intelligence (LNAI), Springer, 2008

\title{
A Fuzzy Controller for Autonomous Negotiation of Stairs by a Mobile Robot with Adjustable Tracks
}

\author{
Winai Chonnaparamutt and Andreas Birk \\ School of Engineering and Science \\ Jacobs University Bremen* \\ Campus Ring 1, D-28759 Bremen, Germany \\ a.birk@iu-bremen.de
}

\begin{abstract}
Tracked mobile robots with adjustable support tracks or flippers are popular promising solutions for negotiating rough terrain and 3D obstacles. Though many according robot bases are in principle physically capable of climbing stairs, it is a non-trivial control-task for a remote tele-operator, especially when the user can not directly see the robot like in search and rescue scenarios. To limit training requirements and to ease the cognitive load on operators, respectively to enable fully autonomous rescue robots, we developed a fuzzy controller for this task, which adjusts the drive forces and the posture of the flipper. The design of the controller is guided by observing the strategies of a trained user when tele-operating a robot with unlimited visual information. In doing so, an Open Dynamics Engine (ODE) simulation of our robot is used where the full set of all physical parameters is accessible for analysis. Based on this data, it is shown in several experiments that the controller is not only capable of climbing stairs but that it does so in a more efficient manner than the human user who served as training model.
\end{abstract}

\section{Introduction}

There are many different options for locomotion systems for land robots, each with its particular pros and cons. Also within search and rescue robotics, many different approaches are used (figure 1), ranging from wheeled over legged to serpentine systems [1][2][3][4][5][6][7]. But tracked locomotion is often considered to be the most versatile locomotion system in difficult environments as it can handle large obstacles and loose soil, hinders and small holes and ditches. Compared to e.g. wheeled system, a tracked system can also develop higher thrust or gross traction force particularly for the operation over weak terrain [8]. This type of locomotion is the most suitable to surmount obstacles, negotiate stairways, and is able to adapt to terrain variations [9][10]. For unstructured environments, e.g., a collapsed building, a construction site, tracked locomotion might hence be the most suitable choice. Accordingly, there are various tracked robots have been employed for hazard missions [11][12][13][14][15].

\footnotetext{
* previously International University Bremen (IUB)
} 


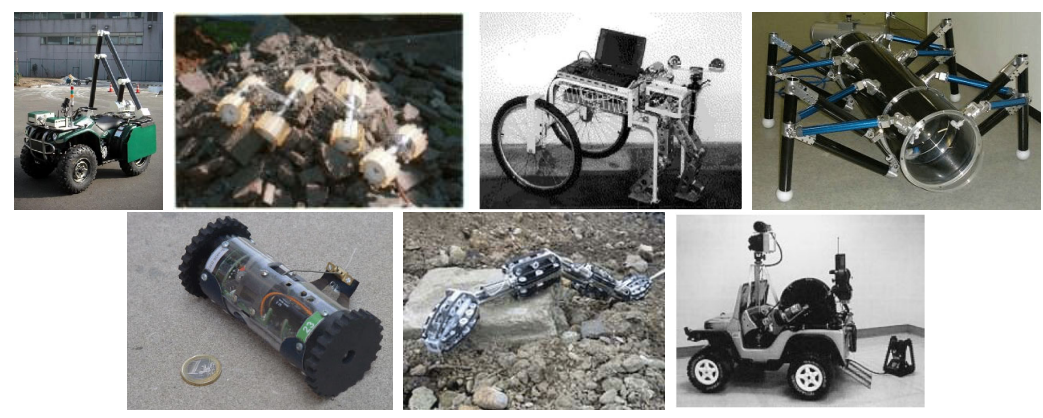

Fig. 1. Examples of the different locomotion approaches for rescue robots: Gryphon-I, Genbu, CUL robot system, Quadruped Jumping Robot, Scout robot, MOIRA, and Marsupial robots [1][2][3][4][5][6][7](from left to right, top to bottom).

But it is almost impossible to select the right parameters for a single pair of tracks. Sometime the footprint of the robot and hence the length of the tracks should be small, for example when negotiating narrow passages. The footprint should on the other hand be large for climbing large obstacles like slopes or stairs. The common solution to this problem is to use additional tracks that can change their posture relative to the main robot body. Examples of variable configuration robots are shown in figure 2. They are successful in many application domains related to rescue robotics [16][17][18] including the RoboCup rescue league [19$21][22-24]$.
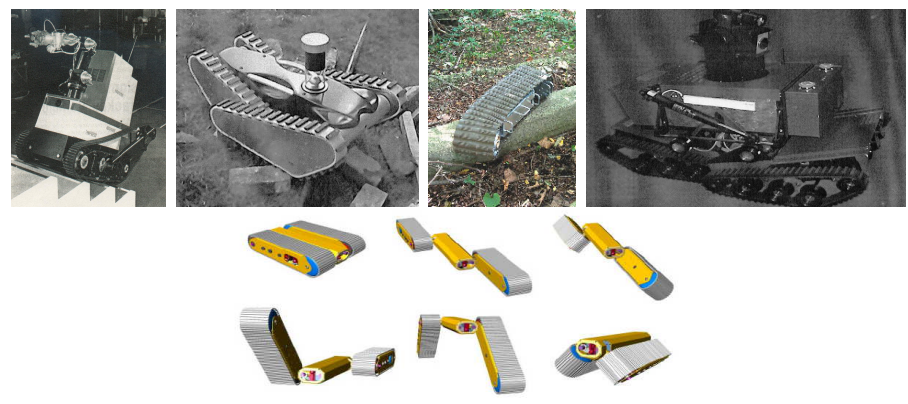

Fig. 2. Variable tracked configuration robots [16]: Pandora [17], AURORA [18], linktype tracked vehicle [25], and NUGV [26]

All aforementioned systems are designed and used for teleoperation. But though these systems are in principle very capable, it is sometimes tremendously hard to negotiate obstacles when the operator can not directly observe the robot. This even holds for commercial systems used in the military domain as indicated by the results of the European Land Robotics Trial 2006 [27]. Intelligent high 
level motion control is hence of interest. An according functionality can be used to relief the operator, respectively to allow for autonomous operations.

\section{The Locomotion System of Jacobs Robotics}
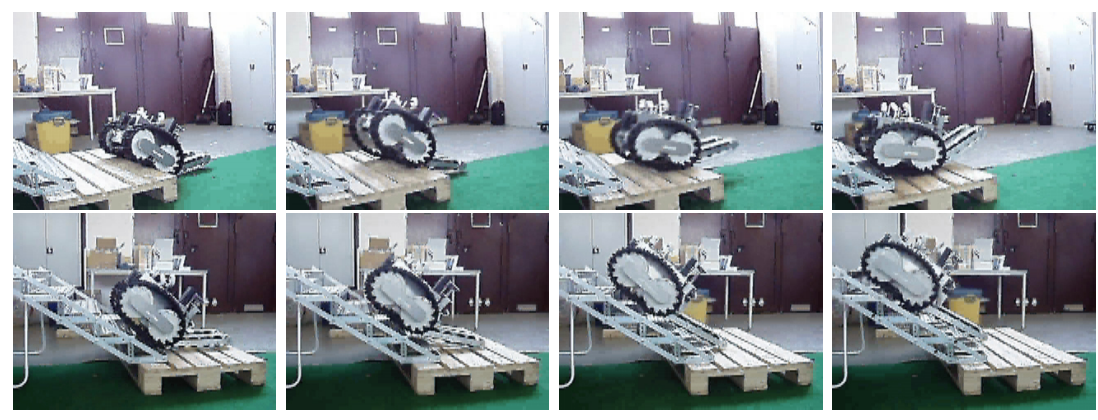

Fig. 3. Rugbot climbing stairs in its teleoperation mode (from left to right, top to bottom).

The work presented here is based on the "rugged robot" or short rugbot platform [28]. It is a variable configuration robot with a special support track or flipper. The flipper mechatronics are based on a special design, which make it particularly robust against shocks and requires smaller joint forces than other state of the art designs. The underlying design and its implementation are described in detail in [29]. Rugbots are capable of climbing stairs (figure 3) and various other obstacles including random step fields. But we also experienced that there is a tremendous difference between "the robot is in principle capable of climbing stairs" and "a remote operator can climb stairs with the robot". The first statement refers to situations where the operator can see the robot from a global perspective much like the view in figure 3 . Then, stair climbing is relatively easy. It is on the other hand much more difficult if this is to be done by a remote operator who is only provided with the sensor views of the robot itself. Hence, autonomous negotiation of complex obstacles is not only of interest for pure fully autonomous operations but also to assist teleoperation.

There are two main disadvantages when doing experiments with autonomous motion control over complex obstacles in the real world. First, crucial properties of the interaction between the system and its environment are very difficult to measure. Especially, contact forces, energy efficiency, etc. are difficult to measure with decent accuracy and meaningful spatial and temporal resolution. Second, experiments in the early testing phase can easily go wrong and pose high risks for the robot as well as the experimenter, e.g., when the robot falls from the stairs. Third, it is very tedious to test the system with various environment parameters, e.g., step widths of stairs, let alone to do this in an exhaustive and 
controlled manner. A commonly used strategy for the design process under such circumstances is hence in general to start with a high fidelity physical simulation.

Here, the Open Dynamics Engine (ODE) [30] is used. Open Dynamics Engine (ODE) is an open source, high performance library for simulating articulated rigid body dynamics, e.g. ground vehicles, legged creatures, and moving objects in virtual environments. ODE is platform independent with an easy to use $\mathrm{C} / \mathrm{C}++\mathrm{API}$. It is designed to be used in interactive or real-time simulation. Its major features are:

- ODE uses a highly stable integrator, so the simulation errors should not grow out of control. ODE emphasizes speed and stability over physical accuracy.

- ODE has hard contacts. This means that a special non-penetration constraint is used whenever two bodies collide.

- ODE has a built-in collision detection system that provides fast identification of potentially intersecting objects, through the concept of spaces.

There are two main components in ODE: a dynamics simulation engine, i.e., world and rigid body, and a collision detection engine, i.e., space and geom (geometry object). The first has the information about the position, velocity, and mass of the rigid body. The latter is given information about the shape of each body. For the first engine, a body is an object which is affected by forces, while a geom of the second engine is an object that can collide with other geoms. A body and a geom together represent all properties of a simulated object.

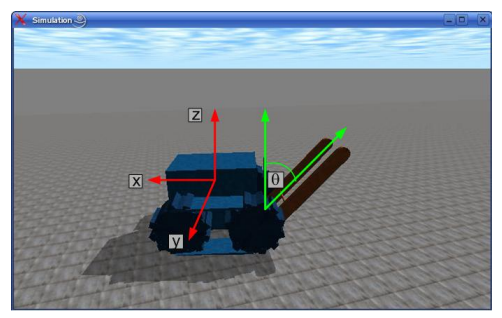

Fig. 4. Rugbot's model in ODE.

The composition of rugbot's body in the simulation are a main body, a flipper ball screw, and four locomotion belts. There are six bodies in the simulation: rugbot main body, four wheel bodies, and a flipper body. Four hinge-2 joints are used for connecting four wheels to the rugbot main body, while the flipper is connected to rugbot via a hinge joint. The overall configuration can be seen in figure 4. To drive a force is applied to the body and torque is applied to the four hinge-2 joints with a fixed speed. The driving force of comes hence from the force on rugbot's body and the torque of the joints. Autonomous driving requires the adjustment of the driving force and of the posture of the flipper. The environment features several obstacles including stairs, ramps and a random step fields (figure 5). 

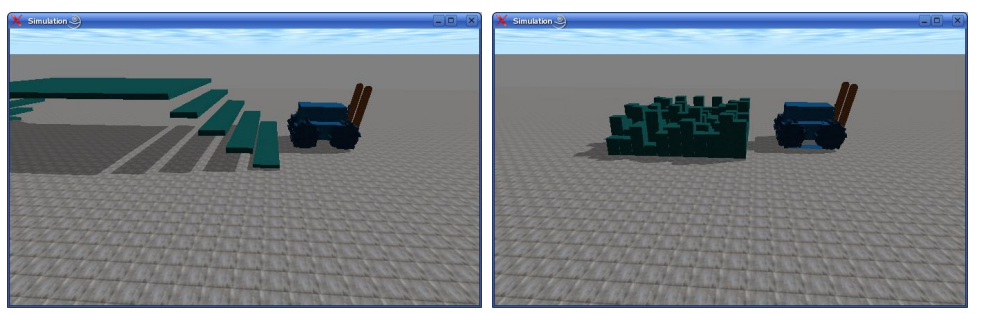

Fig. 5. Examples of obstacles: stairs (left) and a random step field (right).

\section{Mimicking Human Operators}

The intelligent motion controller is separated in two levels: high-level control and low-level control systems. The high-level controller is based on fuzzy logic [31][32], which is a popular choice for this kind of task [33]. The low-level controller is in charge of handling the motors, especially for the flipper. It is based on PID controllers and its behavior has also been intensively studied. Note that the modeling of the robot was done down to a detailed physical simulation of the motor properties. As the low-level controller operates on a different, much faster time scale than the high level controller, it is neglected in the rest of this paper.

The main idea for the design of the high level controller is to observe the control patterns used by a human operator who has "perfect" information about the situation of the robot. These control patterns are then turned into fuzzy control laws, which are autonomously carried out by the robot.

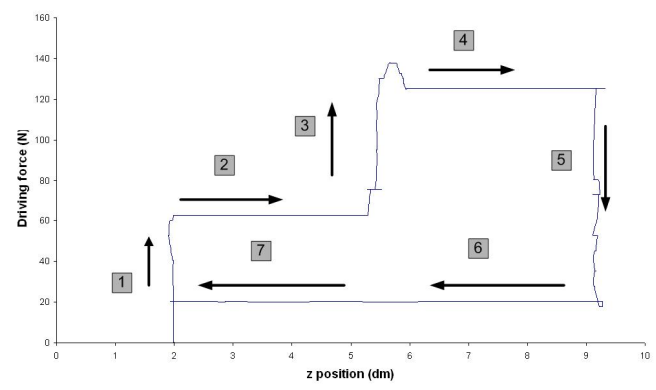

Fig. 6. The relation between the $\mathrm{z}$ position and the driving force as measured in a teleoperation run when climbing stairs; the arrows and numbers indicated the sequence of changes by the operator over time.

Figure 6 shows for example the relation between the $\mathrm{z}$ position and the driving force in a typical teleoperation run when climbing a stair. This type of data was collected over multiple runs. The operator had each time a best possible view, 


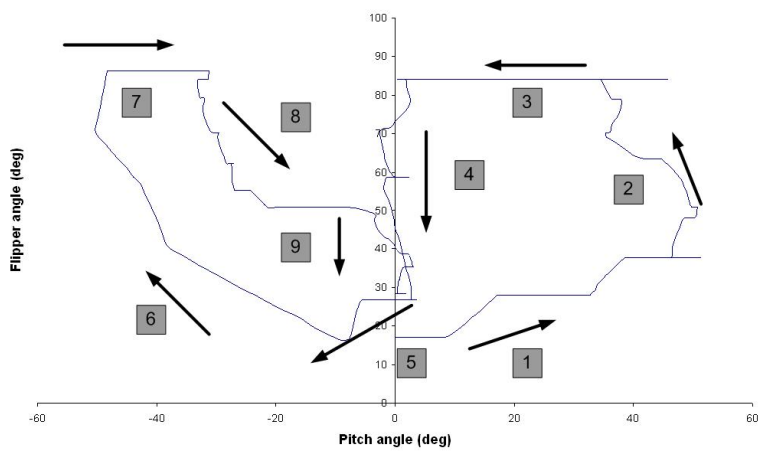

Fig. 7. The relation between the pitch angle and the flipper angle measured in teleoperation run when climbing stairs.

i.e., optimal information about the situation of the robot. Similarly, figure 4 shows the relation between the pitch angle and the flipper angle. This data was then used for designing the high level controller.

The high level controller consists of two fuzzy logic modules, one for the driving force and one for the posture of the flipper. Both run in parallel in the spirit of behavior-oriented control. The fuzzy controller for the driving force was implemented first as it is a prerequisite for designing the fuzzy controller for the flipper.
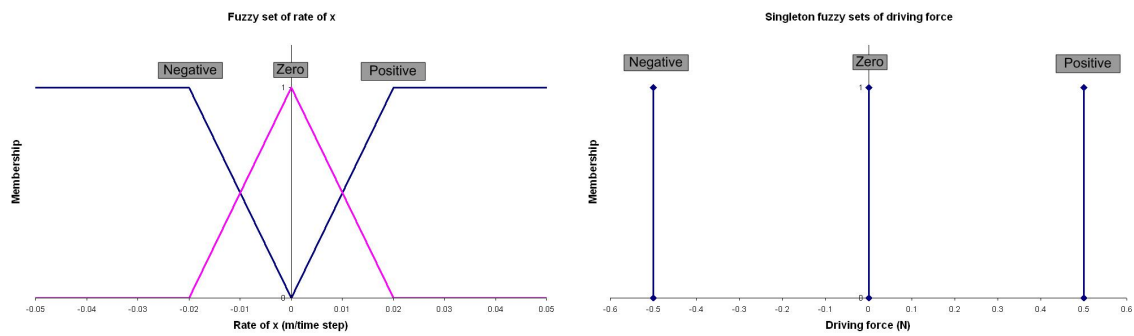

Fig. 8.

Fig. 9. The input fuzzy set of the driving force controller in $x$ direction (left). The output fuzzy set of driving force controller: the rate of driving force (right).

The inputs of the driving force controller are the changing rate of locomotion in $\mathrm{x}$ and $\mathrm{z}$ directions, i.e., $\Delta \mathrm{x}$ and $\Delta \mathrm{z}$. Based on the analyzed data, the input fuzzy sets were defined as shown in figure 9 . The output of the controller is the rate of driving force, $\Delta \mathrm{F}$ (figure 9). Both $\Delta \mathrm{x}$ and $\Delta \mathrm{z}$ have three membership sets each. Each membership also has the same values (unit: $\mathrm{m}$ per sampling 
time): zero, plus at 0.02 , and negative at -0.02 . The rate of driving force also has three membership sets (unit: $\mathrm{N}$ ): zero, plus at 5, and minus at -5 . The fuzzy rules, based on Mamdani fuzzy rules type, are shown in the table 1 The fuzzy inference method to find the output for each rule is based on the Mamdani minimum reference method. An output fuzzy set is converted to be a real number by using center-of-gravity method for singletons [34][35].

\begin{tabular}{|l|l|l|}
\hline$\Delta \mathbf{x}$ & $\Delta \mathbf{y}$ & $\Delta \mathbf{F}$ \\
\hline negative & negative & positive \\
negative & zero & positive \\
negative & positive & positive \\
zero & negative & negative \\
zero & zero & positive \\
zero & positive & positive \\
positive & negative & zero \\
positive & zero & negative \\
positive & positive & zero \\
\hline
\end{tabular}

Table 1. Fuzzy rules for the driving force fuzzy controller
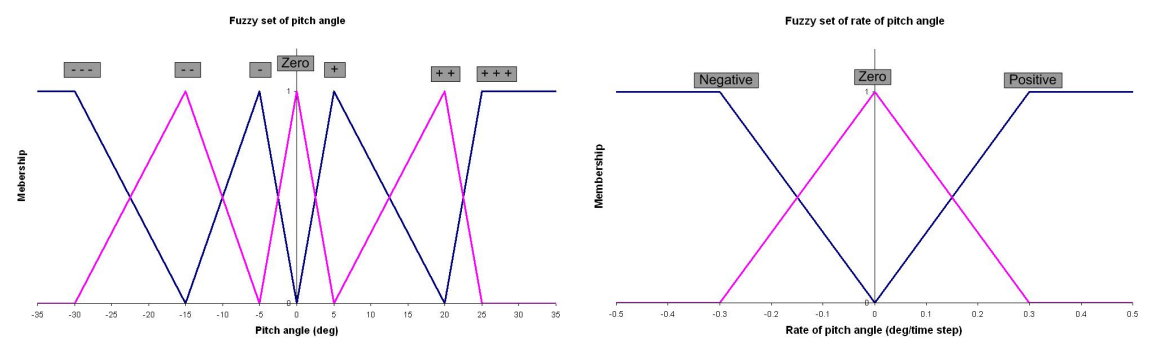

Fig. 10. The input fuzzy sets of the flipper fuzzy controller: the pitch angle of the robot and its rate.

As mentioned before, the second fuzzy logic module of the high level controller adjust the posture of the flipper. It runs in parallel to the drive force controller. The inputs for the flipper fuzzy controller are the pitch angle of Rugbot, $\alpha$, and its rate, $\Delta \alpha$. The output is the rate of the moving angle of the small track relative to the ball screw that drives the mechanism, $\Delta \theta_{F}$. The fuzzy sets of inputs and output are shown in figures 10 and 11 respectively.

The pitch angle has seven membership sets (unit: degree): zero, most plus at $30(+++)$, more plus at $15(++)$, plus with $5(+)$, most negative at $-30(--$ $-)$, more negative at - $15(--)$, and negative at $-5(-)$. The rate of pitch angle has three membership sets (unit: degree per sampling time): zero, plus at 0.3 , and 


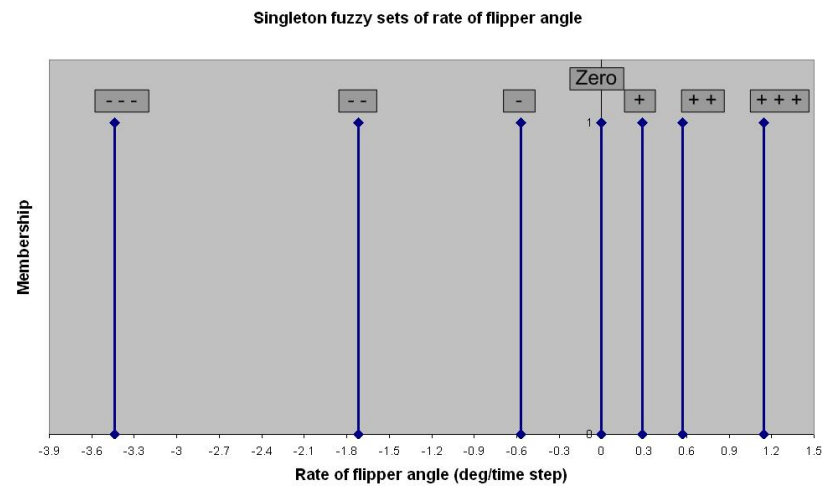

Fig. 11. The output fuzzy set of the flipper fuzzy controller: the rate of flipper angle.

minus -0.3. The rate of flipper angle has seven membership sets (unit: degree per sampling time): zero, most plus at $0.4(+++)$, more plus at $0.2(++)$, plus with $0.1(+)$, most negative at $-1.2(---)$, more negative at $-0.6(--)$, and negative at $-0.2(-)$. Based on Mamdani fuzzy rules type, the fuzzy rules of the controller are defined as in the table 2 :

\begin{tabular}{|c|c|c|c|}
\hline \multicolumn{3}{|c|}{$\Delta \alpha$} \\
\hline & negative & zero & positive \\
\hline \hline$\alpha$ & $\Delta \theta_{F}$ & $\Delta \theta_{F}$ & $\Delta \theta_{F}$ \\
\hline--- & + & + & - \\
-- & ++ & +++ & --- \\
- & zero & zero & zero \\
zero & zero & zero & zero \\
+ & --- & zero & zero \\
++ & zero & + & ++ \\
+++ & zero & + & +++ \\
\hline
\end{tabular}

Table 2. Fuzzy rules for the flipper fuzzy controller

By using the Mamdani minimum reference method for inference and the center-of-gravity method for singletons for deffuzification, we got the results of the controller as shown in figure ??.

\section{The General Performance}

The autonomous controller was intensively tested in various experiments. First and foremost, it is indeed successful in reliably moving the robot stairs up and down without any human intervention. The robot adjusts its driving force and 

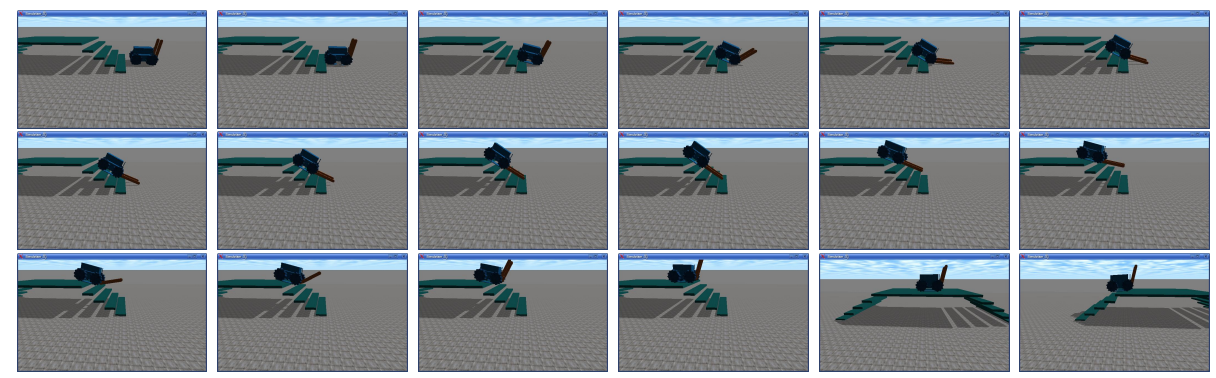

Fig. 12. The autonomous controller moves the robot reliably up on stairs (from left to right, top to bottom).

flipper posture such that its center of gravity is supported, it gets good traction, and that it moves in the desired direction.

Second, it does so more effectively than a human operator does. Concretely, the overall amount of forces, i.e., the energy put into the system, is smaller when the autonomous controller is driving the robot compared to cases when a human operator does exactly the same task. Note that this already holds for the case where the human operator has "perfect" visual information about the robot, i.e., the operator can freely place his viewpoint in the environment. The quantitative amount of this increased effectiveness is of course user and mission dependent. Butthe qualitative properties of this effect can be seen when comparing human control (figures 6 and ) with the autonomous one (figure 13). It can be noticed that the autonomous controller causes much smoother changes. This benefit is also well-known from other applications of fuzzy logic to control.
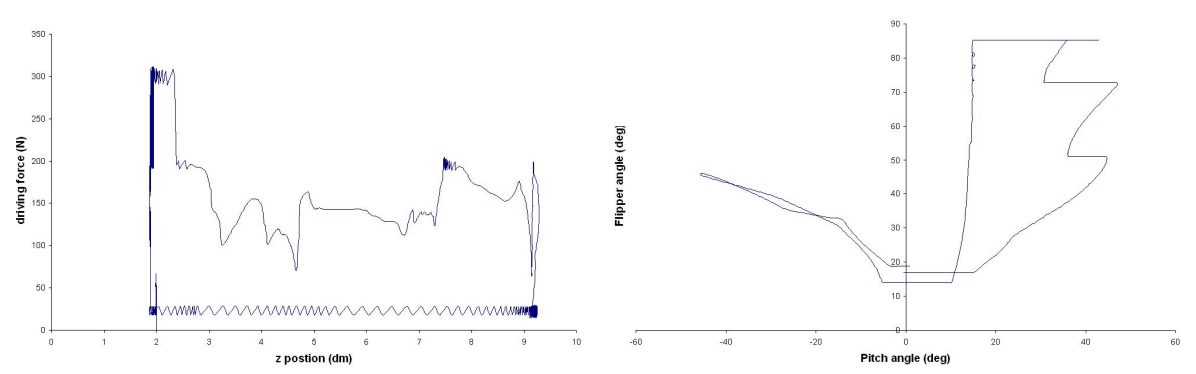

Fig. 13. The relation between the $\mathrm{z}$ position and the driving force when climbing a stair in an autonomous run (left). The relation between the pitch angle of the robot and the flipper angle while driving in autonomous mode (right).

Finally, there are important results with respect to the general usefulness of the controller. It has been designed based on observations of human operator input during tele-operated stair climbing. This is an important, but nevertheless 

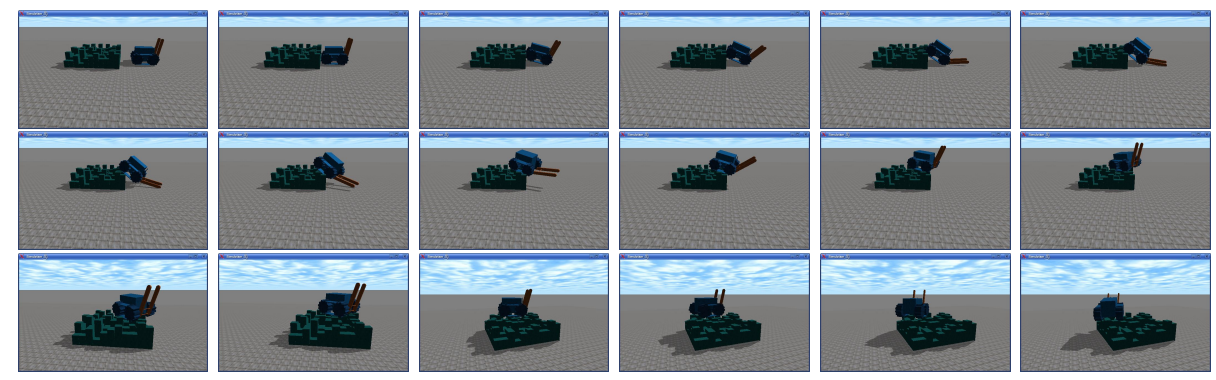

Fig. 14. The controller was designed based on observing the inputs of human operators during stair climbing. But it generalizes to other obstacles. Here, the robot autonomously negotiates a Random Step Field with the controller (from left to right, top to bottom).

very specialized type of locomotion task. On the other hand, the nature of the task comprises of all basic challenges, namely adjusting the center of gravity, making contact with support points for sufficient traction, and adjusting the driving force, which can be found for any other obstacles. And it indeed turned out that the controller does well on other obstacles as well, including ramps and as difficult terrain as modeled by Random Step Fields (figure 14).

\section{Conclusion}

A fuzzy controller for autonomous negotiation of stairs was presented. The main idea for the design of the controller is to observe the control patterns of human operators during tele-operation under optimal conditions, i.e., with "perfect" visual information. The controller is tested in an ODE simulation with a detailed physical model of the robot and various environment parameters. The autonomous controller is first of all capable of driving the robot up and down stairs. Second, it is not only very reliable in doing so, but it outperforms human operators in terms of efficiency. Finally, the autonomous controller seems to generalize very well as it manages to also move the robot autonomously over other obstacles including random step fields.

\section{Acknowledgments}

The authors gratefully acknowledge the financial support of Deutsche Forschungsgemeinschaft (DFG).

Please note the name-change of our institution. The Swiss Jacobs Foundation invests 200 Million Euro in International University Bremen (IUB) over a five-year period starting from 2007. To date this is the largest donation ever given in Europe by a private foundation to a science institution. In appreciation of the benefactors and to further promote the university's unique profile in higher education and research, the boards of IUB have decided to change the university's name to Jacobs University 
Bremen. Hence the two different names and abbreviations for the same institution may be found in this article, especially in the references to previously published material.

\section{References}

1. Debenest, P., Fukushima, E.F., Hirose, S.: Development and control of a buggy robot for operation on unstructured terrain. International Conference on Intelligent Robots and System 1 (2002) 763- 768

2. Kimura, H., Hirose, S.: Development of genbu: Active wheel passive joint articulated mobile robot. International Conference on Intelligent Robots and System 1 (2002) $823-828$

3. Tokuda, K., Osuka, K., Yano, S., Ono, T.: Concept and development of general rescue robot cul. International Conference on Intelligent Robots and Systems 3 (1999) 1902-1907

4. Kikuchi, F., Ota, Y., Hirose, S.: Basic performance experiments for jumping quadruped. International Conference on Intelligent Robots and Systems 3 (2003) $3378-3383$

5. Stoeter, S.A., Rybski, P.E., Gini, M., Papanikolopoulos, N.: Autonomous stairhopping with scout robots. International Conference on Intelligent Robots and System 1 (2002) $721-726$

6. Osuka, K., Kitajima, H.: Development of mobile inspection robot for rescue activities: Moira. International Conference on Intelligent Robots and Systems 3 (2003) $3373-3377$

7. Murphy, R.: Marsupial and shape-shifting robots for urban search and rescue. IEEE Intelligent Systems 15 (2000) 14 - 19

8. Wong, J., Huang, W.: "wheels vs. tracks" - a fundamental evaluation from the traction perspective. Journal of Terramechanics 43 (2006) $27-42$

9. Hardarsson, F.: Locomotion for difficult terrain. Technical Report TRITA-MMK1998:3, Mechatronics Lab, Dept. of Machine Design (1997)

10. Wong, J.Y.: Theory of Ground Vehicles. 3rd edition edn. John Wiley and Sons, Inc. (2001)

11. Inc., R.R.: Houdini: Reconfigurable in-tank robot. Technology Development Data Sheet (1997) $179-180$

12. Isozaki, Y., Nakai, K.: Development of a work robot with a manipulator and a transport robot for neuclear facility emergency preparedness. Advanced Robotic 16(6) (2002) $489-492$

13. Rison, B., Wedeward, K.: Locomotion lecture slide of introduction to design course http://www.ee.nmt.edu/ wedeward/EE382/SP02/locomotion.pdf.

14. Dan, Z., Tianmiao, W., Jianhong, L., Guang, H.: Modularization of miniature tracked reconnaissance robot. Proceedings of the 2004 IEEE International Conference on Robotics and Bioomimetics (2004) 490 - 494

15. Li, B., Ma, S., Liu, J., Wang, Y.: Development of a shape shifting robot for searchand rescue. Proceedings of the 2005 IEEE International Workshop on Safety, Security and Rescue Robotics (2005) $31-25$

16. Iwamoto, T., Yamamoto, H.: Mechanical design of variable configuration tracked vehicle. Journal of Mechanical Design 112 (1990) 289 - 294

17. Schempf, H., Mutschler, E., Piepgras, C., Warwick, J., Chemel, B., Boehmke, S., Crowley, W., Fuchs, R., Guyot, J.: Pandora: autonomous urban robotic reconnaissance system. International Conference on Robotics and Automation, 1999. 3 (1999) $2315-2321$ 
18. Schempf, H.: Aurora - minimalist design for tracked locomotion. Springer Tracts in Advanced Robotics 20 (2003) 453 - 466

19. Lee, W., Kang, S., Kim, M., Shin, K.: Rough terrain negotiable mobile platform with passively adaptive double-tracks and its application to rescue missions. In: Robotics and Automation, 2005. ICRA 2005. Proceedings of the 2005 IEEE International Conference on. (2005) 1591-1596

20. Kang, S., Lee, W., Kim, M., Shin, K.: Robhaz-rescue: rough-terrain negotiable teleoperated mobile robot for rescue mission. In: IEEE International Workshop on Safety, Security and Rescue Robotics, SSRR. (2005) 105-110

21. Lee, W., Kang, S., Kim, M., Park, M.: Robhaz-dt3: teleoperated mobile platform with passively adaptive double-track for hazardous environment applications. In: IEEE/RSJ International Conference on Intelligent Robots and Systems (IROS). Volume 1. (2004) 33-38 vol.1

22. Lee, W., Kang, S., Lee, S., Park, C.: Robocuprescue - robot league team ROBHAZDT3 (south korea). In Noda, I., Jacoff, A., Bredenfeld, A., Takahashi, Y., eds.: RoboCup 2005: Robot Soccer World Cup IX. Lecture Notes in Artificial Intelligence (LNAI). Springer (2006)

23. Kadous, M.W., Kodagoda, S., Paxman, J., Ryan, M., Sammut, C., Sheh, R., Miro, J.V., Zaitseff, J.: Robocuprescue - robot league team CASualty (australia). In Noda, I., Jacoff, A., Bredenfeld, A., Takahashi, Y., eds.: RoboCup 2005: Robot Soccer World Cup IX. Lecture Notes in Artificial Intelligence (LNAI). Springer (2006)

24. Tsubouchi, T., Tanaka, A.: Robocuprescue - robot league team Intelligent Robot Laboratory (japan). In Noda, I., Jacoff, A., Bredenfeld, A., Takahashi, Y., eds.: RoboCup 2005: Robot Soccer World Cup IX. Lecture Notes in Artificial Intelligence (LNAI). Springer (2006)

25. Lee, C.H., Kim, S.H., Kang, S.C., Kim, M.S., Kwak, Y.K.: Double-track mobile robot for hazardous environment applications. Advanced Robotics 17 (2003) 447 $-459$

26. Blackburn, M., Bailey, R., Lytle, B.: Improved mobility in a multidegree-of-freedom unmanned ground vehicle. In: SPIE Proceeding on Unmanned Ground Vehicle Technology VI, 2004, IEEE (2004)

27. ELROB: European land-robot trial (elrob). www.elrob2006.org (2006)

28. Smith, R.: Open dynamics engine http://www.ode.org/.

29. Zadeh, L.A.: Fuzzy sets. Information and Control (1965) 338-353

30. Zadeh, L.A.: Fuzzy logic. IEEE Computer (1988) 83-93

31. Ying, H. In: Fuzzy Control and Modeling. IEEE Press (2000)

32. Jantzen, J.: Design of fuzzy controllers. Technical report, Technical University of Denmark, Department of Automation (1998)

33. Niku, S.B.: 9. In: Introduction to Robotics: Analysis, Systems, Applications. Prentice Hall (2001) 